\title{
Investigação de dosagens da terapia por ondas de choque no tratamento da espasticidade: Um estudo experimental
}

\author{
Investigation of shock wave therapy dosages in the treatment of spasticity: An experimental study \\ Investigación de las dosis de la terapia de ondas de choque en el tratamiento de la espasticidad: un \\ estudio experimental
}

Recebido: 21/09/2021 | Revisado: 02/10/2021 | Aceito: 05/10/2021 | Publicado: 09/10/2021

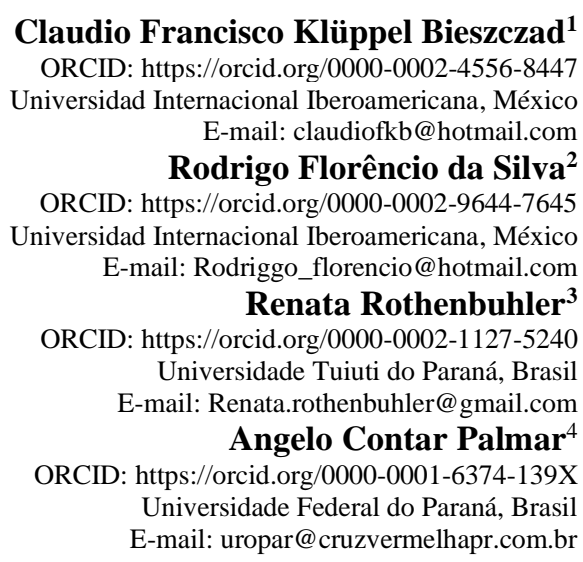

\begin{abstract}
Resumo
Analisa-se as diversas funções das ondas de choque referente à dose, intensidade, tempo de aplicação e a seleção dos pacientes aptos, ou seja, as indicações, contra indicações e suas complicações. Necessita-se aperfeiçoar métodos de aplicação das ondas de choque em espasticidade de maneira segura e coerente. Selecionam-se 23 casos, idade entre 20 a 76 anos. Divididos em dois grupos diferentes. Grupo 1: Composto por 10 indivíduos normais, utiliza-se dosagem elevadas. Dose: $0.050 \mathrm{mj} / \mathrm{mm}^{2}$ a $0,060 \mathrm{mj} / \mathrm{mm}^{2}$. Grupo 2: Composto por 13 indivíduos com espasticidade, utiliza-se as mesmas dosagens elevadas do primeiro grupo para verificar a resistência da pele frente a dosagem. As mensurações compararam os momentos antes e após as aplicações utilizando a escala de Ashworth modificada, goniometria digital, acelerômetro e a inspeção na pele para verificar sinais de hematomas ou petéquias. Ao analisar a média, antes das aplicações o grau de flexão do cotovelo era $(37,2)$ e após as aplicações a flexão do cotovelo evoluiu para $(61,0)$ ficando o cotovelo mais livre e maleável. A segunda articulação que refletiu melhora foi a abdução do ombro. A abdução que apresentava $(40,2)$ passou a apresentar $(49,6)$ graus. A aceleração máxima melhorou comparando os momentos antes e após as aplicações de ondas de choque $(0,97-1,27)$. Não se observa petéquias, eritema e hematomas frente ao equipamento. No final demonstra-se uma tabela com as dosagens e ciclos e energia segura. Conclusão: Houve decréscimo da espasticidade em todas as avaliações. A tolerância dos pacientes ao equipamento foi observada satisfatoriamente nesse estudo.
\end{abstract}

Palavras-chave: Acidente vascular cerebral; Espasticidade; Litotripsia; Modalidades de fisioterapia; Ondas mecânicas.

\footnotetext{
Abstract

${ }^{1}$ Mestre em Gerontologia Social, Doutor em Projetos.

${ }^{2}$ Doutor em Meio Ambiente.

${ }^{3}$ Doutora em Engenharia de Produção.

${ }^{4}$ Mestre em Cirurgia.
}

The different functions of the shock waves regarding dose, intensity, application time and selection of suitable patients are analyzed, that is, the indications, contraindications and their complications. It is necessary to improve methods of applying shock waves in spasticity in a safe and coherent way, 23 cases, aged between 20 and 76 years, are selected. Divided into two different groups. Group 1: Composed of 10 normal individuals, high dosages are used. Dose: $0.050 \mathrm{mj} / \mathrm{mm}^{2}$ to $0.060 \mathrm{mj} / \mathrm{mm}^{2}$. Group 2: Composed of 13 individuals with spasticity, the same high dosages of 
the first group are used to check the skin's resistance to the dosage. The measurements compared the moments before and after applications using the modified Ashworth scale, digital goniometry, accelerometer and skin inspection to check for signs of bruises or petechiae. When analyzing the average, before the applications, the degree of elbow flexion was (37.2) and after the applications, the elbow flexion evolved to (61.0), making the elbow freer and more malleable. The second joint that showed improvement was shoulder abduction. The abduction that presented (40.2) started to present (49.6) degrees. Maximum acceleration improved comparing moments before and after shock wave applications (0.97-1.27). Petechiae, erythema and bruises are not observed in front of the equipment. At the end, a table with dosages and cycles and safe energy is shown. Conclusion: There was a decrease in spasticity in all assessments. The tolerance of patients to the equipment was satisfactorily observed in this study.

Keywords: Cerebrovascular accident; Spasticity; Lithotripsy; Physical therapy modalities; Mechanical waves.

\section{Resumen}

Se analizan las diferentes funciones de las ondas de choque en cuanto a dosis, intensidad, tiempo de aplicación y selección de pacientes idóneos, es decir, las indicaciones, contraindicaciones y sus complicaciones. Es necesario mejorar los métodos de aplicación de ondas de choque en espasticidad de forma segura y coherente. Se seleccionan 23 casos, con edades comprendidas entre 20 y 76 años. Dividido en dos grupos diferentes. Grupo 1: Compuesto por 10 individuos normales, se utilizan dosis elevadas. Dosis: 0,050 mj / mm² a 0,060 $\mathrm{mj} / \mathrm{mm}^{2}$. Grupo 2: Compuesto por 13 individuos con espasticidad, se utilizan las mismas dosis altas del primer grupo para comprobar la resistencia de la piel a la dosis. Las mediciones compararon los momentos antes y después de las aplicaciones utilizando la escala de Ashworth modificada, goniometría digital, acelerómetro e inspección de la piel para detectar signos de hematomas o petequias. Al analizar la media, antes de las aplicaciones, el grado de flexión del codo era $(37,2)$ y después de las aplicaciones, la flexión del codo evolucionó a $(61,0)$, haciendo el codo más libre y maleable. La segunda articulación que mostró mejoría fue la abducción del hombro. La abducción que presentó $(40,2)$ pasó a presentar $(49,6)$ grados. La aceleración máxima mejoró comparando momentos antes y después de las aplicaciones de ondas de choque (0.971.27). No se observan petequias, eritema y hematomas frente al equipo. Al final, se muestra una tabla con dosis y ciclos y energía segura. Conclusión: hubo una disminución de la espasticidad en todas las evaluaciones. En este estudio se observó satisfactoriamente la tolerancia de los pacientes al equipo.

Palabras clave: Accidente cerebrovascular; Espasticidad; Litotricia; Modalidades de fisioterapia; Ondas mecânicas.

\section{Introdução}

Pretende-se analisar a dosagem, tempo de aplicação, intensidade para um tratamento eficaz das ondas de choque em pacientes com espasticidade, para os profissionais poderem utiliza-la de maneira segura e tranquila sem perigo de provocar lesões na pele. Necessita-se aperfeiçoar métodos de aplicação em espasticidade de maneira coerente e efetiva. Esses parâmetros foram argumentados sobre 3 aspetos: 1) Procedimentos corretos ao realizar a terapia por ondas de choque. 2) Segurança ao realizar esses procedimentos. 3) Adequar a dose e intensidade para esse procedimento (Guo, 2017; Picceli 2017; Li 2016).

As ESWT são ondas mecânicas de alta intensidade e de curta duração, geram ondas de choque fora do paciente. As ondas de choque são emitidas em intervalos curtos, centenas de microssegundos aumentando a energia de colapso (Fleckentein, 2017).

Ondas de choque são ondas que se propagam rapidamente em um meio, caracteriza-se por ser um impulso de energia de alta frequência, geradas por vários mecanismos, sendo os principais o eletro-hidráulicos, pizoelétricos e eletromagnéticos. O método mais antigo é o eletrohidráulico, e são geradas por um eletrodo submerso em água (Li 2018; Mattyasovzky, 2018).

As ondas extracorpóreas produzem um fluxo de energia na área tratada medido em $\mathrm{mJmm}^{2}$. A força produzida é controlada por um regulador de voltagem no gerador de partículas. Em relações aos efeitos biológicos da terapia, existe a formação de cavitações e microjatos formando microbolhas no interior dos fluidos, caracterizadas por pressão de > 10 ns com alto pico de pressão de100 mpa e 10 us a duração de seu comprimento de onda. O potencial clinico das ondas de choque são diversos, trata-se de um anti-inflamatório potente, sua ação analgésica é eficaz. Libera moléculas importantes responsáveis por diversas funções orgânicas. O potencial terapêutico dessa energia está relacionado com sua instrumentação e o domínio da mesma (Li 2018; Mattyasovzky, 2018; Yalvaç, 2018). 
As ondas de choque são utilizadas em diversos tratamentos como em pseudoartroses e na tendinose calcária no ombro. No âmbito muscular tem-se verificado que a aplicação da ESWT diminui em grau significativo a espasticidade. Tanto que a evidência confirma que o tônus muscular permanece reduzido por um período, de dose semanas, aproximadamente, cerca de quatro meses. Segundo a Escala de Ashworth utilizada para valorar a espasticidade, um paciente que apresenta uma articulação comprometida, rígida em flexão ou extensão, pode apresentar melhorias no aumento do tônus muscular, seja de forma mais pronunciada ou discreta. A dosagem média utilizada na maioria dos artigos foi de 500 a 1000 ciclos de pulsos em cada músculo em baixa energia (Yalvaç, 2018; Shi, 2017; Kim, 2017).

A segurança do paciente e a precisão médica é importante nesse trabalho, esse tema não foi explorado suficientemente nos diversos estudos. A ultrassonografia, por exemplo, é um procedimento médico que faz uso das ondas sonoras, para fins diagnósticos e terapêuticos, evitando a exposição dos pacientes à radiação ionizante (devido ao fato das ondas sonoras aplicadas não possuírem energia suficiente para ionizar átomos). As complicações mais frequentes após a ESWT são as petequeias (pequenos pontos escuros que aparecem na superfície da pele), ocorrendo de 0,2 a 0,66\% dos pacientes. Acredita-se na possibilidade de dano celular causado pela cavitação gerada pela aplicação das ondas de choque (Li 2018; Mattyasovzky, 2018; Yalvaç, 2018).

A aplicação da energia apropriada interage com a anatomia e o posicionamento do paciente. O direcionamento da energia segue as características anatômicas do paciente, seus órgãos e estruturas ósseas. O posicionamento do indivíduo interage no sucesso da terapia (Guo 2019; Hong 2017).

As repetições do pulso influenciam no tratamento, é relevante não misturar a distância do foco com a profundidade da terapia. O volume focal da corrente extracorpórea influi nos resultados da terapia. Nas ondas de choque radiais a energia se expande por todo o músculo enquanto as ondas de choque focais são direcionadas à um ponto específico dentro do músculo. A quantidade de energia liberada geralmente é medida em milijoules por milímetro ao quadrado, essa energia é quantificada em baixa, média e alta energia. Os ciclos de energia referem-se à quantidade de geração de energia em cada músculo. As características técnicas do litotriptor influenciam nos riscos do paciente. Os intervalos entre pulsos influenciam nos danos teciduais. As ondas extracorpóreas podem produzir lesões leves na pele algumas vezes (Li 2018; Mattyasovzky, 2018; Yalvaç, 2018).

Os hospitais e clínicas especializadas em ESWT possuem equipamentos capazes de diminuir a espasticidade muscular. Localizam-se nas dependências das clínicas urológicas e hospitais com infraestrutura adequada para o atendimento desses pacientes. Os pacientes devem ser acompanhados em toda a fase do tratamento. O tratamento é realizado com o paciente deitado, sem a necessidade de anestesia. Os aparelhos são equipados com um sistema duplo de raios-x (diagnóstico por imagem) e ultrassom computadorizado, fazendo com que a terapia seja realizada em alta precisão (Guo 2019; Zhang 2019; Kang 2017; Lou 2017).

O aparelho utilizado em ondas de choque é o mesmo utilizado para fragmentar cálculos renais. A ESWT é um método minimamente invasivo. Nas ondas de choque o litotridor produz a energia fora do indivíduo, atravessando o corpo. Em qualquer procedimento terapêutico, a ESWT possui riscos potenciais e complicações, a segurança deve ser conhecida pelos operadores destes equipamentos (Zhong 2019; Shi 2017; Kang 2017).

Os artigos científicos têm procurado comprovar a redução da espasticidade ao utilizar ondas de choque. Os valores da dosagem divergem entre si. Alguns pesquisadores com medo de causar danos aos pacientes utilizam valores extremamente baixos. Os artigos com dosagem próxima a $0,060 \mathrm{mj} / \mathrm{mm}^{2}$ são os melhores. Portanto, deve-se fazer uma seleção dos artigos mais efetivos. A comunidade científica, os fisioterapeutas e médicos possuirão maior segurança ao utilizarem um protocolo seguro de tratamento (Zhong 2019; Radinmehr 2017; Taheri 2017; Tsung-Ying 2016). 


\section{Método}

O experimento foi realizado no hospital da Cruz Vermelha no departamento uropar. Este trabalho foi submetido ao número de aprovação do projeto no comitê de ética em pesquisa: 48495115.0.0000.0093. Os participantes foram informados e esclarecidos a respeito do estudo e de seus objetivos e procedimentos. Os voluntários que participaram do estudo assinaram os termos de consentimento livre e esclarecido.

\section{Amostra}

A amostra compõe-se de 23 casos com idade entre 20 a 76 anos. Os indivíduos com espasticidade apresentam idade entre 56 a 76 anos ( \pm 66 anos). Os indivíduos normais apresentam idade entre 20 a 50 anos. Foram divididos em dois grupos diferentes.

\section{Caracterização do estudo}

O estudo foi dividido em dois grupos diferentes. Grupo 1: Composto por 10 indivíduos normais, utiliza-se dosagens elevadas de ondas de choque. Dose: $0.050 \mathrm{mj} / \mathrm{mm}^{2}$ a $0,060 \mathrm{mj} / \mathrm{mm}^{2}$. Grupo 2: Composto por 13 indivíduos com espasticidade, utiliza-se as mesmas dosagens elevadas do primeiro grupo. Dose: $0,050 \mathrm{mj} / \mathrm{mm}^{2}$ a $0,060 \mathrm{mj} / \mathrm{mm}^{2}$. O objetivo foi observar a redução da espasticidade e elucidar alguns aspectos em relação as ondas de choque, como as alterações na pele. Foram realizadas uma aplicação em cada voluntário (Wu 2018; Yoon 2017). O suporte metodológico para essa pesquisa baseia-se nos experimentos de Yoon, S. et al. (2017).

Nos indivíduos normais os critérios de inclusão são 1) Saúde adequada. 2) Idade entre 20 a 50 anos. 3) ausência de AVC e dificuldades motoras. Os critérios de exclusão são: 1) Saúde inadequada. 2) Distúrbios do movimento. 3) Uso de anticoagulante. Os critérios de inclusão dos pacientes são: 1) Espasticidade moderada. 2) Espasticidade severa. Os critérios de exclusão são: 1) Espasticidade leve. 2) Deformidades. 3) Limitação articular. 4) Uso de anticoagulante.

\section{Análise estatística}

A amostra foi composta de 23 casos, dez demonstraram espasticidade severa e três demonstraram espasticidade moderada. Os indivíduos normais apresentaram idade entre 20 a 50 anos. As variáveis quantitativas foram descritas pelas medidas de média, mediana, valores mínimo e máximo e desvio padrão para comparação dos dois momentos de avaliação em relação a variáveis que não atenderam a condição de normalidade. O objetivo foi comparar os momentos antes e após as aplicações para a média das mensurações dos três métodos. Utilizou-se assim o teste T-Student Pareado pela escala de Ashworth Modificada e o teste de Kolmogorov-Smirnov (KS) para as demais mensurações.

\section{Instrumentos de coletas de dados}

O aparelho utilizado é a Direx integra ${ }^{\circledR}$, nas mensurações foram utilizados quatro métodos. A escala de Ashworth, acelerômetro, goniometria digital e a inspeção da pele. A escala de Ashworth, validada pelo instituto nacional de saúde em AVC (NIHSS), foi utilizada como critério de comparação.

\section{Ondas de choque}


Define se como dosagem elevada 0,060mj/mm² e dosagem baixa 0,030mj/mm² (Zhong 2019; Li 2018; Kang 2017; Li 2016). A dosagem de $0,060 \mathrm{mj} / \mathrm{mm}^{2}$ são elevadas, porém, são interpretadas como não invasivas, o resultado das aplicações são raramente algumas petéquias ( $\mathrm{Li} 2016)$.

O aparelho de ESWT foi a Direx, integra ${ }^{\circledR}$, gerador de energia eletromagnética, foi escolhido 1000 ciclos por segundo e $0,030 \mathrm{mj} / \mathrm{mm}^{2} 1$ energia em $12 \mathrm{gpm}$. Buscou-se observar a diminuição da espasticidade através de uma dose/intensidade segura.

\section{Escala de Ashworth}

As pessoas foram examinadas pela escala de Ashworth modificada, descrita por Bohmann (1987), trata-se de um teste subjetivo para verificar os níveis de espasticidade. A escala de Ashworth foi validada pelo Instituto Nacional de Saúde em AVC (NIHSS), são medidas clínicas para a espasticidade sendo utilizadas por fisioterapeutas e pesquisadores (Bohmann 1987; Ashworth 1964).

\section{Goniômetro digital}

Em seguida avalia-se os pacientes pelo goniômetro digital (medidor de ângulo digital $10^{\circ}$, mensuração de 0 a 360 graus, resolução 0,05, Precisão 0,1 grau) cujo os procedimentos foram: 1) Marcar os pontos anatômicos. 2) Captar os dados. 3) Registrar os dados (Mori 2017; Wang 2016).

\section{Acelerômetro}

Em terceiro lugar foi utilizado um acelerômetro em que os sinais de contração muscular, captado pelo acelerômetro, estão em três eixos $\mathrm{x}, \mathrm{y}$ e $\mathrm{z}$, formando 3 curvas de acordo com cada eixo, utilizou-se um equipamento BMA254, com sensor da marca Bosch. O acelerômetro capta vibrações mecânicas musculares, essas vibrações são colocadas no computador em forma de gráfico. Esse gráfico mensura o deslocamento do ventre muscular. É uma técnica não invasiva que registra as vibrações produzidas pelos músculos ao se contrair. Essas vibrações também são chamadas de oscilações. Os procedimentos foram: 1) Avaliar a fadiga muscular. 2) Diagnosticar vibrações cardíacas. 3) Analisar o potencial evocado (Xiang 2018; Intiso 2017). Aplicou-se uma corrente elétrica aussie que produziu uma contração muscular, tornando possível captar os sinais do acelelômetro. Quanto maior o deslocamento do ventre muscular produzido pelo acelerômetro, menor a espasticidade presente, pois seus músculos estão mais saudáveis (Wu 2018).

\section{Inspeção}

Foi realizada uma inspeção na pele dos voluntários para verificar o grau de dano muscular frente a aplicação do equipamento. Durante a análise do ventre muscular foram observados os seguintes parâmetros: 1. Eritema. 2 . Petéquias. 3. Hematômas.

\section{Posicionamento dos voluntários ao realizar os procedimentos}

Posição: Os voluntários devem estar deitados em decúbito dorsal. O antebraço deve estar em uma posição estendida o máximo possível. O antebraço deve estar perfeitamente encostado na bolha em que irá ser irradiada as ondas de choque, utiliza-se um gel de contato entre bolha e o músculo.

Aplicação: Durante a aplicação das ondas de choque, o braço e o antebraço devem estar alongados, para que a energia se espalhe por toda a superfície muscular. A aplicação dura 10 minutos.

Abaixo apresenta-se um fluxograma demonstrando de maneira sequencial as etapas do experimento. 
Tabela 1 - Fluxograma do estudo descrevendo a sequência do experimento. Aplica-se as quatro formas de mensuração: Escala de Ashworth modificada, goniometria digital, acelerômetro e a inspeção da pele.

\begin{tabular}{|l|l|}
\hline \multicolumn{2}{|c|}{ Ordem sequencial da metodologia } \\
\hline $\begin{array}{l}\text { Avaliação } \\
\text { inicial }\end{array}$ & $\begin{array}{l}\text { Escala de Ashworth modificada - Grau da hipertonia } \\
\text { Goniometria digital - Amplitude do movimento } \\
\text { Acelerômetro - Deslocamento do ventre muscular } \\
\text { Inspeção da pele }\end{array}$ \\
\hline $\begin{array}{l}\text { Ondas } \\
\text { choque }\end{array}$ & Baixa intensidade \\
\hline $\begin{array}{l}\text { Avaliação } \\
\text { final }\end{array}$ & $\begin{array}{l}\text { Escala de Ashworth modificada - Grau da hipertonia } \\
\text { Goniometria digital - Amplitude do movimento } \\
\text { Acelerômetro - Deslocamento do ventre muscular } \\
\text { Inspeção da pele }\end{array}$ \\
\hline
\end{tabular}

Fonte: Elaboração própria.

\section{Resultados}

O grupo 1 foi formado pelos indivíduos normais, a intensidade das ondas de choque variou entre $0,050 \mathrm{mj} / \mathrm{mm}^{2}$ a $0,60 \mathrm{mj} / \mathrm{mm}^{2}$, após as aplicações a pele permaneceu normal, durante a inspeção não foram observados eritema, petéquias e hematomas frente ao equipamento. As mesmas intensidades foram aplicadas no grupo 2 onde também não foram observadas alterações na pele. Além de sua espasticidade reduzir eficazmente. Ao utilizar a escala de aswhorth modificada e o Teste TStudent Pareado na figura abaixo, destacam-se seis pacientes que obtiveram melhoras significativas, sendo o (valor $\mathrm{p}<0,001)$ na escala de Ashworth. Apenas dois pacientes não melhoraram, os possíveis motivos serão analisadas na discussão. Segundo a Escala de Ashworth modificada, quanto maior o valor apresentado mais elevada a espasticidade desse paciente (Bohannon 1987). Por exemplo: espasticidade grau 4 significa que o paciente apresenta uma espasticidade severa. Quando a escala apresenta grau 1 significa que a espasticidade é leve ou grau 0 no qual o paciente não possui espasticidade. De acordo com o gráfico abaixo, em todas as quatro barras a espasticidade diminuiu. Na flexão do braço, onde a espasticidade apresentava $(3,15)$ passou a apresentar após as aplicações $(1,77)$. Em todas as demais comparações houve decréscimo do componente espástico. $\mathrm{O}$ valor $\mathrm{p}$ foi significativo em todas as variáveis $(<0,001)$. 
Figura 1 - Compara os dois momentos para a mensuração através da escala de Ashworth modificada. Verifica-se os parâmetros flexão do braço, extensão do braço, abdução do braço e flexão do cotovelo antes e após as aplicações das ondas de choque. Observa-se evolução na flexão do braço $(3,15-1,77)$. Valor p $(<0,001)$.

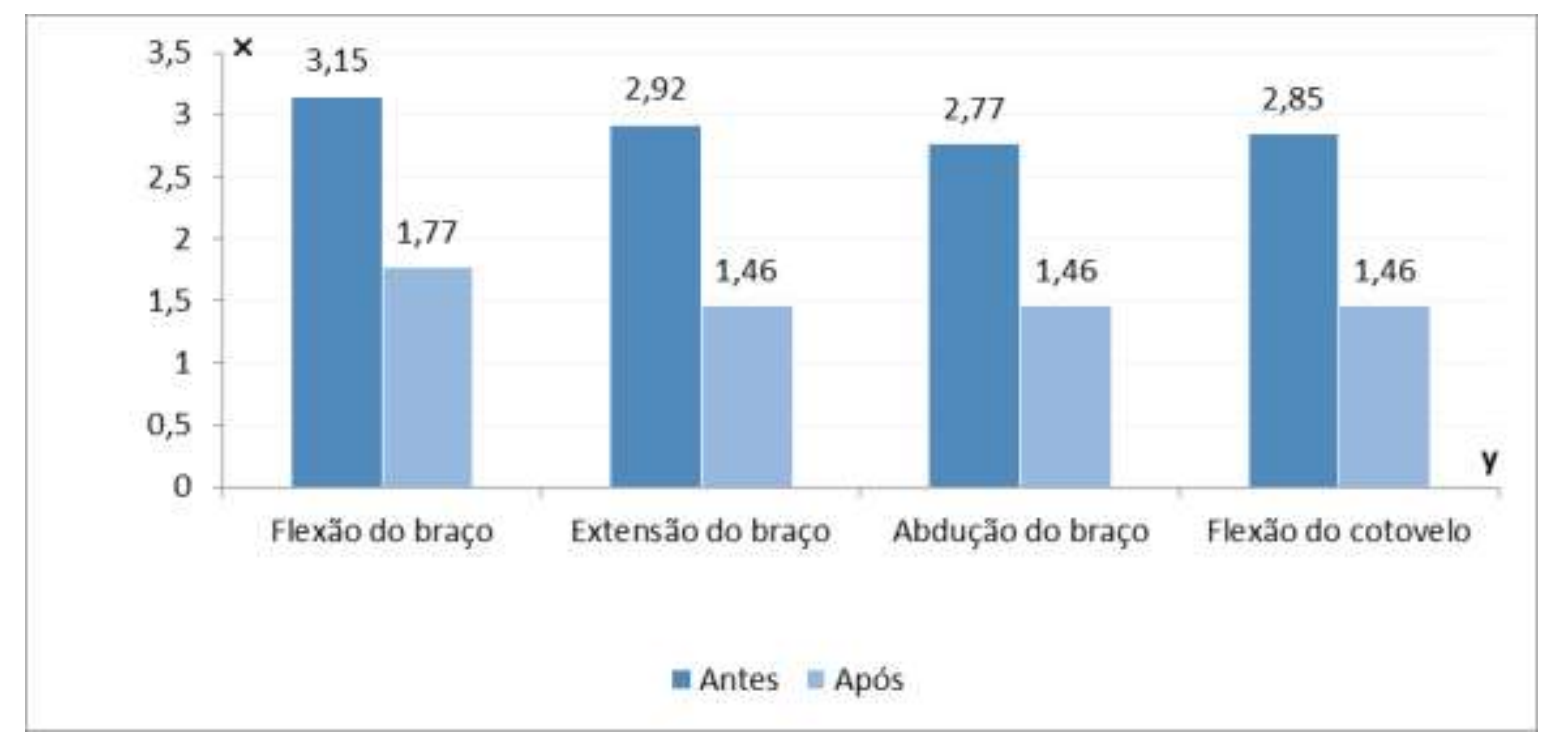

Teste T-Student Pareado. Fonte: Resultados da pesquisa.

Figura 2 - Compara os dois momentos para a mensuração do goniômetro digital. O valor p corresponde respectivamente: flexão do ombro $(<0,001)$; extensão do ombro: $(0,034)$; abdução do ombro $(<0,001)$ e flexão do cotovelo: $(>0,005)$, antes e após as aplicações das ondas de choque. A flexão do cotovelo aumentou $(37,2-61,0)$. As demais variáveis demonstram melhoras significativas.

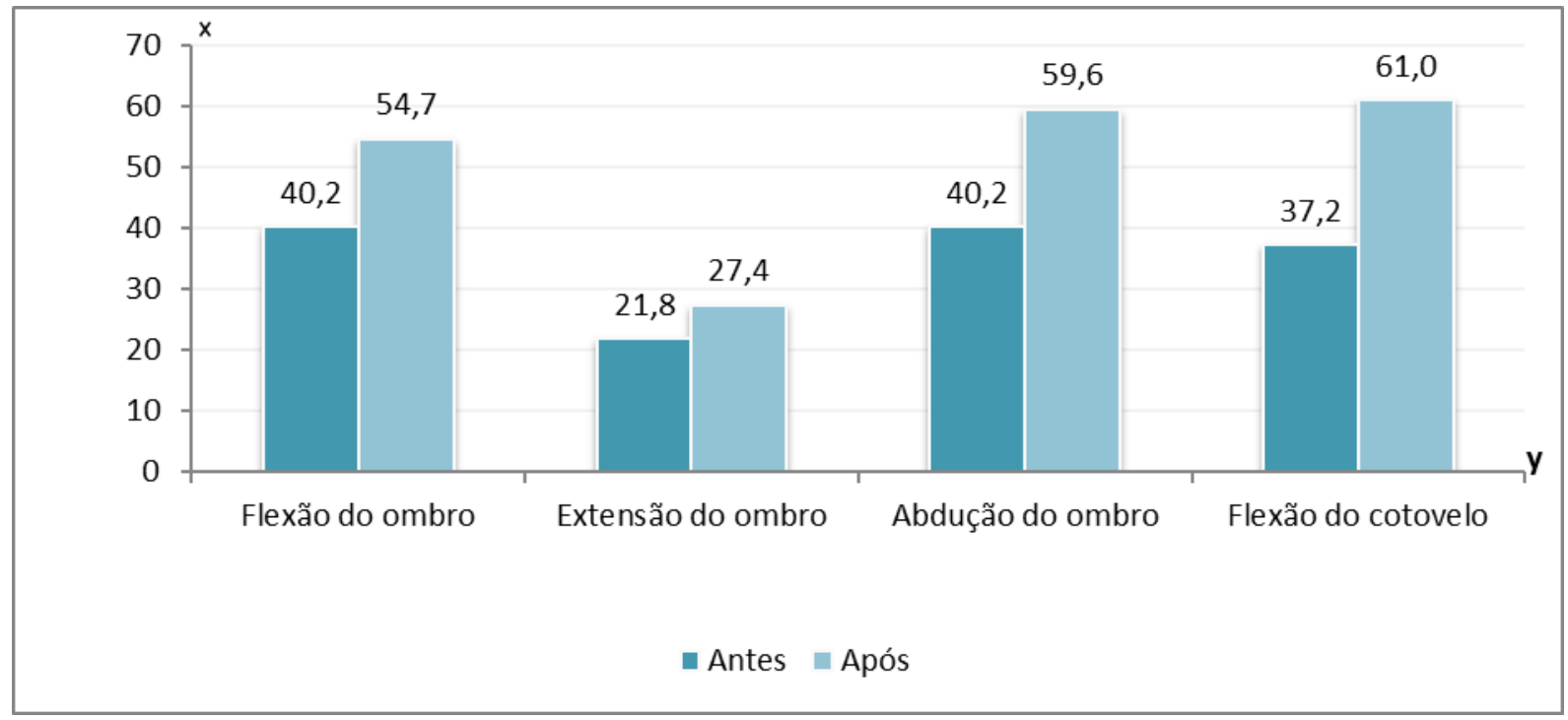

Teste Kolmogorov-Smirnov (KS). Fonte: Resultados da pesquisa.

Comparando momentos para a goniometria digital utilizando o Teste Kolmogorov-Smirnov acima citado os resultados foram favoráveis. Durante a flexão do cotovelo os resultados foram muitos significativos $(37,2-61,0)$, ficando o cotovelo mais livre e maleável. Assim acontece nas demais articulações. Apenas a extensão do ombro evoluiu pouco, (21,8-27.4), tendo pouca significância. A maioria dos músculos do corpo humano são biarticulares ou triarticulares. As articulações são entrelaçadas por músculos, portanto quando libera-se a articulação, igualmente reflete-se na liberação articular. A segunda articulação que refletiu melhora foi a abdução do ombro, onde apresentava $(40,2)$ e passou a apresentar $(49,6)$ graus. Em 
relação aos desvios padrões a flexão do ombro houve variação (21,0-33,7), a flexão do ombro variou um ponto apenas (21.620.6), a extensão do ombro que anteriormente sua média não houve significância, seu desvio padrão foi (10.8-18-4), a abdução do ombro houve variação (21,3-26.6).

O parâmetro flexão do ombro não houve significância (p valor =0,034). O valor p é superior a significância adotada ( $>>0,005)$. Em todas as outras variáveis houve significância, flexão do ombro $(<0,001)$; abdução do ombro $(<0,001)$ e flexão do cotovelo: $(>0,005)$.

Figura 3 - Compara os dois momentos para a mensuração do acelerômetro. O valor p corresponde respectivamente: tempo de dilatação: $(0,003)$; aceleração máxima $(<0,001)$ e tempo de aceleração $(0,128)$, antes e após as aplicações das ondas de choque. Neste parâmetro o tempo de dilatação houve melhora de (0,22-0.08) milissegundos. A aceleração máxima melhorou $(0,97-$ $1,27)$.

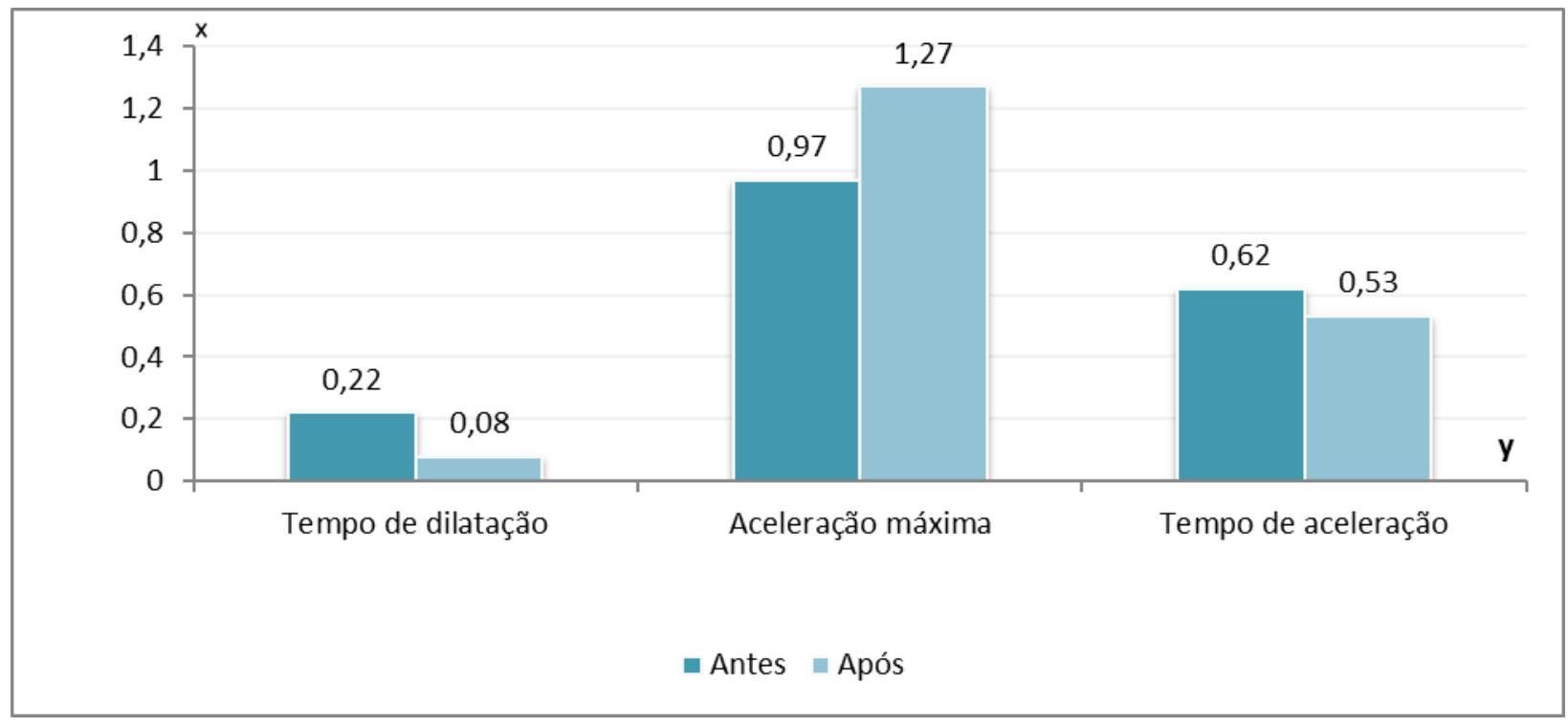

Teste Kolmogorov-Smirnov (KS). Fonte: Resultados da pesquisa.

Segundo a acelerometria (KS) as variáveis tempo de dilatação e tempo de aceleração, quanto maior o valor melhor a evolução do paciente. Por exemplo: O tempo de dilatação reflete o tempo que inicia a contração muscular, portanto quanto mais curto o tempo melhor a contração muscular. Nos parâmetros citados na figura acima o tempo de dilatação melhorou, de $(0,22)$ passou a um tempo de $(0.08)$ milissegundos. O tempo de aceleração reflete o tempo gasto para realizar uma contração muscular completa. Quando o musculo é patológico com espasticidade, o tempo de contração é maior. Quando o músculo é normal seu tempo de contração é mais rápido. Na variável tempo de aceleração, quando o tempo de aceleração é menor o resultado é melhor. A aceleração máxima melhorou comparando os momentos antes e após as aplicações de ondas de choque $(0,97-1,27)$.

O valor $\mathrm{p}$ corresponde respectivamente: tempo de dilatação: $(0,003)$ para o decréscimo: $(0,22-0,08)$; aceleração máxima $(<0,001)$ para o aumento: $(0,97-1,27)$ e tempo de aceleração $(0,128)$ não houve significância.

A Tabela 2 demonstra os critérios avaliados nas aplicações das ondas de choque. Verificou-se que a dosagem foi eficiente para reduzir a espasticidade de modo eficaz. A dosagem apresenta segurança quando aplicada abaixo de 1000 ciclos por minutos. Alguns pacientes apresentaram algumas petéquias. Próximo aos 1000 ciclos os pacientes referem sentir dor no local aplicado. A dose e a intensidade também devem ser consideradas, porém, os ciclos de energia fornecem realmente o diferencial durante as aplicações. 
Nos grupos musculares grandes, como os descritos acima, a energia foi significativa em 700 a 1000 ciclos por segundo. Enquanto os músculos pequenos, no qual, não estão descritos acima, ficam livres para utilizar energias mais baixas.

Considera-se a dose/intensidade normal a frequência de 0,030 a $0,060 \mathrm{mj} / \mathrm{mm}^{2}$. Neste trabalho foram investigadas as doses mais elevadas. Essas doses elevadas podem ser utilizadas sem nenhum problema, porém ressalta-se o cuidado de não focar o eletrodo acima do osso porque o paciente refere dor. Embora existam diversas pesquisas sobre a utilização das ondas de choque no crescimento ósseo (Shi 2017). O tempo de duração dos efeitos da ESWT sobre a espasticidade foi de aproximadamente 12 semanas.

Apresenta-se abaixo algumas sugestões de aplicação de ondas de choque baseada nesse estudo. A pesquisa demonstrou que a faixa de 700 a 1000 ciclos em cada músculo em uma energia próximo a $0,060 \mathrm{mj} / \mathrm{mm}^{2}$ não causa danos ao paciente e reduz a espasticidade satisfatoriamente.

Tabela 2 - Tabela demonstrando sugestões de aplicação de acordo com os critérios avaliados nos grupos musculares flexores, extensores, abdutores do braço e flexores do cotovelo. A dosagem apresenta segurança quando aplicada abaixo de 1000 (7001000) ciclos em cada músculo em uma dose de 0,050 a $0,060 \mathrm{mj} / \mathrm{mm}^{2}$.

\begin{tabular}{llll}
\hline & \multicolumn{2}{l}{ Demonstração dos critérios de aplicação } & Ciclos \\
\hline Grupos Musculares & Músculos envolvidos & Dose $\left.\mathbf{( m j} / \mathbf{m m}^{\mathbf{2}}\right)$ & \\
\hline & & & 700 \\
Flexores do braço & Deltoide, bíceps & 0,050 a 0,060 & 700 \\
Extensores do braço & Tríceps, deltoide & 0,050 a 0,060 & 700 \\
Abdutores do braço & Deltoide, infraespinhal & 0,050 a 0,060 & 700 \\
Flexores do cotovelo & Bíceps, braquiorradial & 0,050 a 0,060 & \\
\hline
\end{tabular}

Fonte: Resultados da pesquisa.

\section{Discussão}

O efeito da ESWT durou dose semanas aproximadamente, houve diminuição do tônus muscular nos pacientes com espasticidade. A espasticidade aumentou aos poucos nessas doses semanas permitindo que houvesse tempo para a intervenção da fisioterapia, proporcionando um resultado melhor e permanente (Taheri 2017).

A tolerância dos pacientes ao equipamento foi observada satisfatoriamente nesse trabalho. A espasticidade foi reduzida satisfatoriamente de IV para II segundo a escala de Ashworth modificada, e permaneceu reduzido por um período de dose semanas (Ashworth 1964; Bohannon 1967). A corrente extracorpórea em espasticidade pode produzir irritações leve na pele algumas vezes, em todos os casos as irritações são muito leves. As petéquias muito raramente apareceram. Em diversos artigos não relata-se aparecimento de petéquias nem eritema, apenas uma leve coloração avermelhada (Guo 2017; Yoon 2017). Muitos pesquisadores utilizam dosagens baixas com medo de causarem algum eventual danos aos pacientes. Geralmente a dosagem é baseada em artigos publicados. As doses são experimentais as vezes baixas, as vezes altas (Li 2016; Radinmehr 2017; Taheri 2017).

A influência da energia extracorpórea nas células e organelas celulares foram analisados no cordão umbilical humano cujo pulso chegou a 2.000 ciclos. A diminuição do padrão espástico e a resistência da energia no corpo humano, formaram um resultado global daquilo que se pretendeu investigar, que são os critérios de aplicação das ondas de choque, e a formação de um protocolo que servirá como guia para futuras aplicações (Yalvaç 2018; Maro 2017; Shi 2017). 
Necessário acentuar que a aplicação da ESWT interage com a anatomia e o posicionamento do paciente. O direcionamento da energia deve seguir as características anatômicas do paciente, seus órgãos e estruturas ósseas. O posicionamento do indivíduo é muito importante para o sucesso desta modalidade de tratamento (Li 2016).

A disponibilidade e os custos do equipamento para realizar as ondas de choque limitam a quantidade de pacientes em que se pretende estudar. Existe limitação quando queremos aplicar a energia extracorpórea em um universo grande de voluntários. Neste trabalho foi realizada uma aplicação em cada voluntário, devido às limitações de acesso ao equipamento e o desgaste dos eletrodos, extremamente caros, foram realizadas apenas uma aplicação das ondas de choque em cada voluntário.

A espasticidade conduz a deformidades articulares, e muita dor provocada pelo encurtamento (Arredondo 2016). O tratamento é sempre aplicado em baixa intensidade (Zhang 2019). As diversas pesquisas não entram em um real consenso referente à dose, intensidade, tempo de aplicação e a seleção dos pacientes aptos, ou seja, as indicações e contra indicações e suas complicações. A participação da fisioterapia também é importante no êxito do tratamento, em trabalhos futuros pode-se incluir pesquisas com a ESWT em conjunto com a fisioterapia. Necessita-se aperfeiçoar os métodos de aplicação da energia extracorpórea em espasticidade de maneira coerente e efetiva. Deve-se buscar uma inserção nos tratamentos de saúde como um novo procedimento médico ou fisioterapêutico (Li 2018).

A dosagem e intensidade segura foi concluída. Essa dosagem, ao ser aplicada novamente, chegaram a resultados similares. Foram solucionadas as indicações e as limitações clínicas das ondas de choque para a satisfação dos pacientes e do corpo clínico. Verificou-se as condições que influenciam o decréscimo da espasticidade (Wu 2018; Xiang 2018; Ashworth 1964).

\section{Considerações Finais}

A tolerância ao equipamento foi observada, a ESWT pode ser aplicada nos parâmetros sugeridos. Essa nova modalidade de tratamento representa um avanço no tratamento da espasticidade. As ondas de choque têm sido aproveitadas em diversas áreas da saúde. Futuramente esse tratamento será mais usual e os diversos ângulos existentes serão explorados de forma aprofundada. A reabilitação neurofuncional também está incluída neste contesto (Li 2018; Wu; 2018; Guo 2017).

\section{Referências}

Arredondo A P, Ramires E C, \& Mora P C. (2016). Baclofen in the therapeutic of sequele of traumatic brain injury: spasticity. Clin neuropharmacol, 39(6):311-315.

Ashworth B. (1964). Preliminary of trial corisodoprol in multiple sclerosis. NP, 2:192-520.

Bohannon R W, \& Smith M B. (1987). Interrater reability of modified Ashworth scale of muscle spasticity. Phys Ther, 67(2):206-207.

Fleckenstein, J., Friton, M., Himmelreich, H., \& Banzer, W. (2017). Effect of a single administration of focused extracorporeal shock wave in the relief of delayed-onset muscle soreness: results of a partially blinded randomized controlled trial. Archives of physical medicine and rehabilitation, 98(5), 923-930.

Guo P, Goo F Zao T, Sun W, \& Wang B. (2017). Positive effect of extracorporeal shock wave therapy on spasticity in post stroke patients: a meta analysis. $J$ Stroke Cerebrovasc Dis, 26(11):2470-2476.

Guo, J., Qian, S., Wang, Y., \& Xu, A. (2019). Clinical study of combined mirror and extracorporeal shock wave therapy on upper limb spasticity in poststroke patients. International jornal of rehabilitation research internationale zeitschrift fur Rehabilitationsforschung. Revue internationale de recherches de readaptation, $42(1), 31$.

Hong, J. O., Park, J. S., Jeon, D. G., Yoon, W. H., \& Park, J. H. (2017). Extracorporeal shock wave therapy versus trigger point injection in the treatment of myofascial pain syndrome in the quadratus lumborum. Annals of rehabilitation medicine, 41(4), 582.

Intiso, D., Santamato, A., \& Di Rienzo, F. (2017). Effect of electrical stimulation as an adjunct to botulinum toxin type A in the treatment of adult spasticity: a systematic review. Disability and rehabilitation, 39(21), 2123-2133.

Kim, Y. W., Chang, W. H., Kim, N. Y., Kwon, J. B., \& Lee, S. C. (2017). Effect of extracorporeal shock wave therapy on hamstring tightness in healthy subjects: a pilot study. Yonsei medical journal, 58(3), 644-649. 
Kang, N., Zhang, J., Yu, X., \& Ma, Y. (2017). Radial extracorporeal shock wave therapy improves cerebral blood flow and neurological function in a rat model of cerebral ischemia. American journal of translational research, 9(4), 2000.

Kang N. Zhang J, Yu X, \& Ma Y. (2017). Radial extracorporeal therapy improves cerebral blood flow and neurological function in a rat model of cerebral ischemia. Am J Transl Res, 9(4) 2000-2012.

Li T, M D, Chang C, M D, Chou Y, Ph D, Chen L, Chu H, M D, Chiang S, Chang S, \& Wu Y. (2016). Effect of Radial Shock WaveTherapy on Spasticity of the Upper Limb in Patients With Chronic Stroke, Medicine (Baltimore), 95(18):e3544.

Li D, Robin H A, Cleveland O,\& J'erusalem A. (2018). 3D multicellular model of shock wave-cell interaction. Acta biomater, 41(4):1742-7061.

Lou J, Wang S, Liu S, \& Xing G. (2017). Effectiviness of extracorporeal shock wave therapy whithout local anesthesia in patients: a meta-analysis of randomized controlled trials. Am J Phys Med Rehab, 96(8):529-534.

Mattyasovszky, S. G., Langendorf, E. K., Ritz, U., Schmitz, C., Schmidtmann, I., Nowak, T. E., \& Drees, P. (2018). Exposure to radial extracorporeal shock waves modulates viability and gene expression of human skeletal muscle cells: a controlled in vitro study. Journal of orthopaedic surgery and research, 13(1), 75 .

Maro A, Leo A, Russo M, Casella C, \& Buda, A. (2017). Breakhoughs in thespasticity manangement: are non-pharmacological treatments the future. J Clin Neurosci, 39(16)27.

Mori, L., Marinelli, L., Pelosin, E., Gambaro, M., Trentini, R., Abbruzzese, G., \& Trompetto, C. (2017). Radial shock wave therapy: effect on pain and motor performance in a paralympic athlete. European journal of physical and rehabilitation medicine, 53(2), 286-289.

Picceli A, Marchina E, Gajofatto F, Pontilllo A, Vangelista A, \& Fillippina R. (2017). A sonographic and clinical effect of botulinum toxin type A combined with extracorporeal shock wave therapy on spastic muscle of children with cerebral palsy. Dev Neurohabil, 20(3):160-164.

Radinmehr H, Ansari N, Naghdi S, Obiali G, \&Tabatabari A. (2017). Effect of one session radial extracorporeal shock wave therapy on post stroke plantar flexor spasticity a single-blind clinical trial. Desabil Rehabil, 39(5):483-490.

Shi, L., Gao, F., Sun, W., Wang, B., Guo, W., Cheng, L., ... \& Wang, W. (2017). Short-term effects of extracorporeal shock wave therapy on bone mineral density in postmenopausal osteoporotic patients. Osteoporosis International, 28(10), 2945-2953.

Taheri P, Vahdatpour B, Mellat M, Ashtari F, \& Akbari M. (2017). Effect of Extracorporeal Shock Wave Therapy on Lower Limb Spasticity in Stroke Patients. Arch Iran Med, 20(6):338 - 343.

Tsung-Ying L. Chih-Ya C, Yu-Ching C, Liang-Cheng C, Heng Y C, Shang-Ling C, Shin-Isu C, \& Yung-Tsan W. (2016). Effect of radial shock wave therapy on spasticity of the upper limb in patients with chonic stroke. Medicine (Baltimore), 95(18)e3544.

Wang T, Dir L, Shan L, Dong H, Feng J, Kiessling M C, Angslman N B, Schmitz C, \&Jia F. (2016). A prospective Case-control study of radial extracorporeal shock wave therapy for spastic plantar flexor muscle in very yong children with cerebral palsy. Medicine(Baltimore), 95(19)e3649.

Wu R Y, Chang C N, Chen Y M, \& Hu G C. (2018). Comparasion of effect of focused and radial ewtracorporeal shock waves spastic equinus patients with stroke a randomized controlled trial. Euro j phys rehabil med 92(2):2-35.

Wu, Y. T., Yu, H. K., Chen, L. R., Chang, C. N., Chen, Y. M., \& Hu, G. C. (2018). Extracorporeal Shock Waves Versus Botulinum Toxin Type A in the Treatment of Poststroke Upper Limb Spasticity: A Randomized Noninferiority Trial. Archives of physical medicine and rehabilitation, 99(11), $2143-2150$.

Xiang, J., Wang, W., Jiang, W., \& Qian, Q. (2018). Effects of extracorporeal shock wave therapy on spasticity in post-stroke patients: a systematic review and meta-analysis of randomized controlled trials. Journal of rehabilitation medicine, 50(10), 852-859.

Yalvaç B, Mesci N, Külcü D G, \& Yurdakul O V. (2018). Comparison of ultrasound and extracorporeal shock wave therapy in lateral epicondylosis. Acta orthopaedica et traumatologica turcica, 52(5), 357-362.

Yoon S, M D, Shin M K, Choi E J, \& Kang H J. (2017). Effective Site for the Application of Extracorporeal Shock-Wave Therapy on Spasticity in Chronic Stroke: Muscle Belly or Myotendinous Junction. Ann Rehabil Med, 41(4):547-555.

Zhang, Z. X., Zhang, D., Yu, X. T., \& Ma, \& Y. W. (2019). Efficacy of radial extracorporeal shock wave therapy for chronic pelvic pain syndrome: A nonrandomized controlled trial. American journal of men's health, 13(1), 1557988318814663.

Zhong Z, Liu B, Liu G, Chen J, Li Y, Chen J, \& Hu, Y. (2019). A randomized controlled trial on the effects of low-dose extracorporeal shock wave therapy in patients with knee osteoarthritis. Archives of physical medicine and rehabilitation, 10, 1-31. 